\title{
Superluminal motion in a compact steep spectrum radio source 3C 138
}

\author{
Z.-Q. Shen ${ }^{1}$, D. R. Jiang ${ }^{2}$, S. Kameno ${ }^{3}$, and Y. J. Chen ${ }^{2}$ \\ 1 Institute of Space and Astronautical Science, Yoshinodai 3-1-1, Sagamihara, Kanagawa 229-8510, Japan \\ 2 Shanghai Observatory, Shanghai 200030, PR China \\ e-mail: djiang, cyj@center.shao.ac.cn \\ 3 National Astronomical Observatory, Osawa 2-21-1, Mitaka, Tokyo 181-8588, Japan \\ e-mail: kameno@hotaka.mtk.nao.ac.jp
}

Received 5 December 2000 / Accepted 31 January 2001

\begin{abstract}
We present the results of $5 \mathrm{GHz}$ VLBI observations of a compact steep spectrum source 3C 138. The data are consistent with the western end being the location of the central activity. The observed offset between different frequencies in the central region of 3C 138 can be accounted for by a frequency dependent shift of the synchrotron self-absorbed core. Our new measurements confirm the existence of a superluminal motion, but its apparent velocity of $3.3 \mathrm{c}$ is three times slower than the reported one. This value is consistent with the absence of parsec-scale counter-jet emission in the inner region, but seems still too high to allow the overall counter-jet to be seen in terms of Doppler boosting of an intrinsically identical jet. Either an interaction of jet with central dense medium, or an intrinsically asymmetrical jet must be invoked to reconcile the detected superluminal speed with the observed large scale asymmetry in 3C 138.
\end{abstract}

Key words. radio continuum: galaxies - galaxies: active - galaxies: compact - galaxies: jets - galaxies: nuclei quasars: individual: $3 \mathrm{C} 138$

\section{Introduction}

The radio source $3 \mathrm{C} 138(=4 \mathrm{C} 16.12=\mathrm{J} 0521+1638)$ is identified with a quasar with $m_{\mathrm{v}}=18.84$ and $z=0.759$ (Hewitt \& Burbidge 1989). It is a compact, powerful quasar with a well-defined turn-over in the spectrum at about $100 \mathrm{MHz}$ and a steep high frequency spectrum of $0.65\left(S \propto \nu^{-\alpha}\right)$, making it a prototype of the compact steep spectrum (CSS) radio sources (Fanti et al. 1990). Its spectrum remains straight up to $22 \mathrm{GHz}$, supporting that no dominant flat spectrum core exists (Kameno et al. 1995).

The radio structure of $3 \mathrm{C} 138$ consists of a core with a bright jet and compact lobe to the east and a fainter, more diffuse lobe to the west from the VLA and MERLIN observations (Akujor et al. 1993 and references therein; Lüdke et al. 1998). High resolution VLBI observations (Geldzahler et al. 1984; Fanti et al. 1989; Nan et al. 1992; Dallacasa et al. 1995; Cotton et al. 1997) reveal several knots within the main jet emission extending about 400 mas in a position angle of $65^{\circ}$ and a low brightness counter-jet about 250 mas away in the opposite direction. There are two knots dominating the central 10 mas region. Fanti et al. (1989) claimed on the basis of apparent

Send offprint requests to: Z.-Q. Shen, e-mail: zshen@vsop.isas.ac.jp size that the eastern knot is the core. Cotton et al. (1997) argued that the western one is the location of the nuclear core mainly because of its very weak polarization $(<0.4 \%)$ compared to a peak polarized intensity of $3.5 \%$ for the eastern component at $5.0 \mathrm{GHz}$. Cotton et al. (1997) also

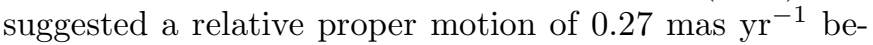
tween the two knots, corresponding to an apparent velocity of $9.7 \mathrm{c}$ (assuming $H_{0}=65 \mathrm{~km} \mathrm{~s}^{-1} \mathrm{Mpc}^{-1}$ and $q_{0}=0.5$, hereafter).

In general, the radio emission from CSS sources is thought to be relatively free of beaming effects, consistent with the fact that CSS sources are the least variable of compact extragalactic radio sources, and CSS sources are rarely found to be superluminal (O'Dea 1998). When combined with the observed two-sided jet structure, such a superluminal motion in $3 \mathrm{C} 138$, if confirmed, will strictly confine the jet velocity and its direction to the line of sight of the observer. We report on 5.0 GHz VLBI observations of $3 \mathrm{C} 138$ as an attempt to confirm its proper motion and probe its compact radio core area.

\section{Observations and data reduction}

The VLBI observations were carried out at $5.0 \mathrm{GHz}$ on November 8, 1997 with the enlarged European VLBI Network composed of Effelsberg (100-m), Hartebeesthoek 


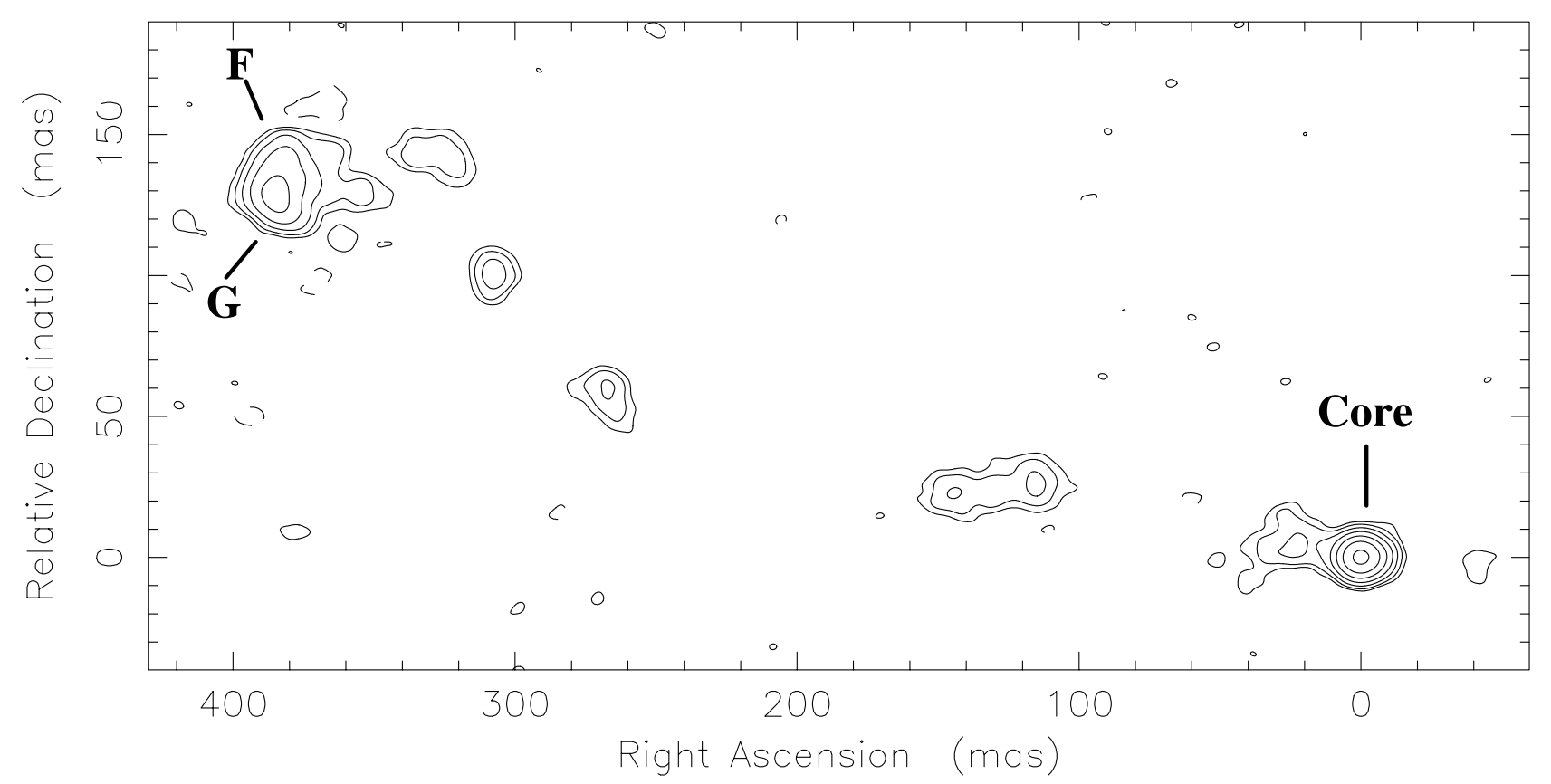

Fig. 1. Tapered $5 \mathrm{GHz}$ VLBI image of 3C 138 restored with a 10 mas circular beam. Contour levels are drawn at -3.6 , $3.6 \times 2^{n}$ mJy beam $^{-1}(n=0,1, \ldots, 6)$. The peak flux density is $274 \mathrm{mJy}_{\text {beam }}{ }^{-1}$. The rms noise is $1.2 \mathrm{mJy} \mathrm{beam}^{-1}$

(26-m), Jodrell MK2 (25-m), Medicina (32-m), Noto (32-m), Onsala (25-m), Shanghai (25-m), Torun (32-m), Urumqi (25-m) and Westerbork (25-m). Sources were observed in a snapshot mode, i.e., 5-12 thirteen-minute scans spreading across a wide range in hour angle to obtain a good $\mathrm{u}-\mathrm{v}$ coverage. The left-circular polarized radio signals were recorded in Mark III mode $\mathrm{C}$ with a total bandwidth of $14 \mathrm{MHz}$ (7 IFs and $2 \mathrm{MHz}$ per IF band) at each station. The correlation of the data was performed on the MPIfR MK III Correlator in Bonn, Germany with an output averaging time of $4 \mathrm{~s}$.

Then, a priori visibility amplitude calibrations for each antenna were applied using the antenna gain and the system temperatures measured during the observations. After the data were globally fringe-fitted in AIPS (Schwab \& Cotton 1983), the subsequent data inspection, imaging and modelfitting were carried out with DIFMAP (Shepherd 1997).

Because of the large separation ( 400 mas) between the eastern compact lobe and central core region in 3C 138, special attention was paid to the smearing effects of time averaging and bandwidth in order to image an undistorted full field. The $2 \mathrm{MHz}$ data in each IF were not averaged through $7 \mathrm{IFs}$, and the averaging time was only $12 \mathrm{~s}$ to minimize smearing effects.

Images were produced with the usual iterations of cleaning and phase self-calibration. Only a constant gain factor for each IF band of each antenna was implemented in the later stage of imaging. These gain corrections are consistent with those obtained for other sources (Shen et al. in preparation). To improve the sensitivity to the low surface brightness regions, the visibility data were naturally weighted and tapered with $F W H M=30 M \lambda$.

\section{Results}

\subsection{Overall structure}

The overall morphology of $3 \mathrm{C} 138$, as shown in Fig. 1, resembles past VLBI images. There are two notable emission regions: the central core and the eastern lobe, which are separated by 400 mas at a position angle of $70^{\circ}$. In between them are several discrete emission components.

The total cleaned flux density in the image (Fig. 1) is $\sim 0.85 \mathrm{Jy}$, which is the same as the correlated flux density on the shortest baseline, Westerbork-Jodrell MK2, but far less than the values of 3.24 Jy and 3.58 Jy by Dallacasa et al. (1995) and Cotton et al. (1997), respectively. The single-dish measurements from the University of Michigan Radio Astronomy Observatory (UMRAO) show a very stable total flux density of $3.80 \mathrm{Jy}$ at $4.8 \mathrm{GHz}$ with a variation less than $6 \%$ over past 10 years. The source is heavily resolved on baselines longer than $3 M \lambda$ (see Fig. 2c in Fanti et al. 1989). So, the main reason for the shortage of cleaned flux density in our image is the lack of shorter baselines like MERLIN or VLA-Pie Town (cf. Dallacasa et al. 1995). As a result, the more extended, lower surface brightness regions seen in previous images (e.g. Fanti et al. 1989) are not well recovered in our image, which causes discontinuity in the main jet emission towards the eastern lobe. The connection of separated emissions (including the core region), however, exhibits a sign of oscillation seen from other observations. No counter-jet was significantly detected in our image though there are some very weak diffuse emissions at about 3 times noise level around the counter-jet region of the $1.7 \mathrm{GHz}$ image by Cotton et al. (1997). The rms noise in the image is $1.2 \mathrm{mJy} /$ beam, 


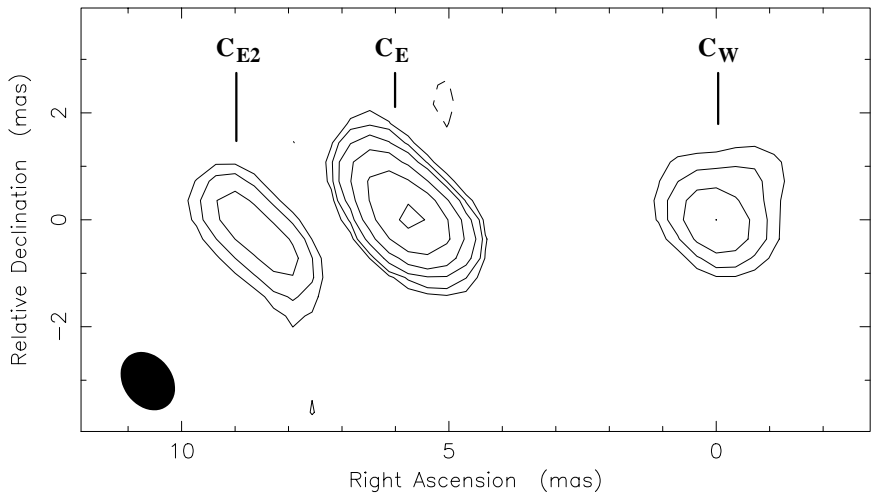

Fig. 2. Full resolution $5 \mathrm{GHz}$ VLBI image of the central core region of $3 \mathrm{C} 138$. The resolution is 1.2 mas $\times 0.92$ mas at a position angle of $37^{\circ}$ (indicated at the lower left corner). Contour levels are drawn at $-3.6,3.6 \times 2^{n} \mathrm{mJy}_{\text {beam }}{ }^{-1}(n=0$, $1, \ldots, 5)$. The peak flux density is $134 \mathrm{mJy}^{\text {beam }}{ }^{-1}$. The rms noise is $1.2 \mathrm{mJy}^{\text {beam }}{ }^{-1}$

about twice the expected thermal noise for the total onsource time of $1.3 \mathrm{hr}$.

Two compact components (labeled F and G after Fanti et al. 1989) in the eastern lobe can be clearly seen. They are aligned nearly orthogonal to the jet axis, which may be interpreted as a working surface of the jet due to its interaction with the surrounding medium.

\subsection{Central core region}

For the purpose of identifying the center of activity, a full resolution image is displayed in Fig. 2. This image was made from the same calibrated data as Fig. 1, except that the baselines shorter than $30 M \lambda$ were removed and the uniform weighting was adopted to ensure a high resolution. To yield a quantitative description, modelfitting to both amplitudes and phases in the calibrated visibility data was applied. Because of the complicated overall structure and the limited observational data, we fit a model to core region only while keeping the cleaned components for the remaining structure (cf. Piner et al. 2000). In this way, we can minimize the effect mainly from the components $\mathrm{F}$ and $\mathrm{G}$. We also tried a modelfitting to the longer baselines data corresponding to Fig. 2. We even made an image of the central region only with a 1-minute averaging time and then fit the visibility data. All these results are quite consistent. In Table 1 is presented the best fitting model consisting of three circular Gaussian components.

The sizes of components $C_{\mathrm{E}}$ and $C_{\mathrm{W}}$ are about the same, but $C_{\mathrm{E}}$ is much brighter. The flux density of $C_{\mathrm{E}}$ is stable over past 12 years (cf. Akujor et al. 1993; Dallacasa et al. 1995). The two point spectral indices $\left(\alpha_{5.0 \mathrm{GHz}}^{8.6 \mathrm{GHz}}\right)$ obtained by combining the high resolution VLBA results at 8.6 GHz (Fey \& Charlot 1997) are almost the same for both $C_{\mathrm{E}}$ and $C_{\mathrm{W}}$ (see Table 1 ). Component $C_{\mathrm{E} 2}$ is less well constrained because it is much too resolved. Actually, $C_{\mathrm{E} 2}$ emission has appeared in several previous $5 \mathrm{GHz}$ images
Table 1. Gaussian model for the central region of 3C 138

\begin{tabular}{lrrrccc}
\hline Component $S /[\mathrm{Jy}]$ & $r /[\mathrm{mas}]$ & $\theta /\left[^{\circ}\right]$ & $a /[\mathrm{mas}]$ & $\alpha_{5.0 \mathrm{GHz}}^{8.6 \mathrm{GHz}}\left(S \propto \nu^{-\alpha}\right)$ \\
\hline$C_{\mathrm{E}} \ldots \ldots$ & 0.218 & -5.76 & 90.0 & 0.768 & 0.46 \\
$C_{\mathrm{E} 2}$ & $\ldots$ & 0.051 & -8.75 & 91.7 & 1.320 & 0.45 \\
$C_{\mathrm{W}}$ & $\ldots \ldots$ & 0.051 & 0 & 0 & 0.753 & 0.45 \\
\hline
\end{tabular}

S: the flux density in Jy; $(r, \theta)$ : the distance and position angle of each component with respect to component $C_{\mathrm{W}}$ in mas and degrees, respectively; $a$ : the diameter (FWHM) of circular Gaussian component in mas; $\alpha_{5.0}^{8.6 \mathrm{GHz}}$ : two point spectral index

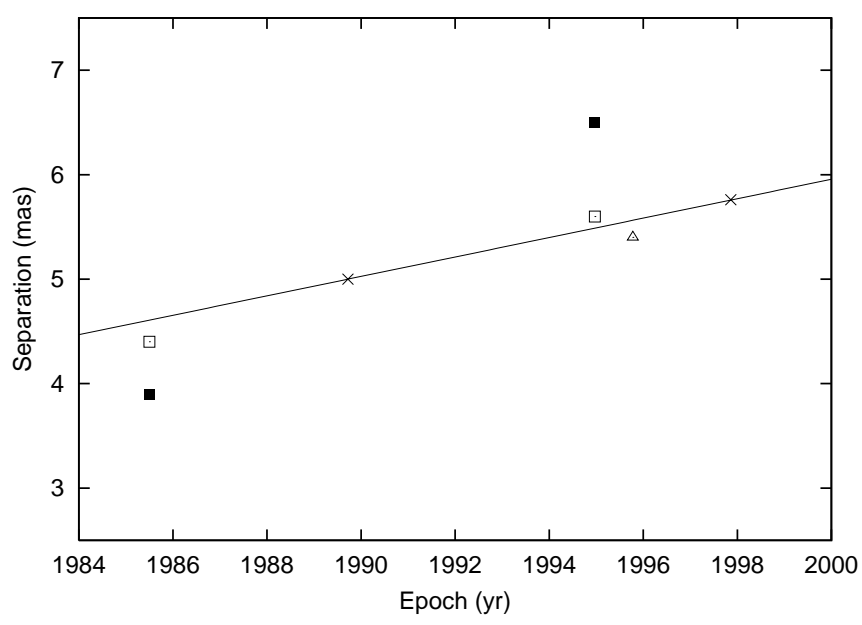

Fig. 3. The apparent separation of two central components $C_{\mathrm{W}}$ and $C_{\mathrm{E}}$ in $3 \mathrm{C} 138$ as a function of the observing epoch. These measurements are made at $5.0 \mathrm{GHz}$ except one (denoted by a open triangle) at $8.6 \mathrm{GHz}$. Symbols are explained in the text. Also shown is a fitting line (see text)

(e.g. Fig. 13 in Cotton et al. 1997), but was first fitted at 8.6 $\mathrm{GHz}$ by Fey \& Charlot (1997).

\section{Discussion}

\subsection{Proper motion}

There are three published $5 \mathrm{GHz}$ VLBI measurements made at epochs 1985.5 (Fanti et al. 1989), 1989.72 (Dallacasa et al. 1995) and 1994.97 (Cotton et al. 1997). Fanti et al. (1989) resolved the central region with two compact components, but did not present the separation at the time. Dallacasa et al. (1995) made the first measurement of the apparent separation of 5.0 mas. Cotton et al. (1997) estimated the separation of 3.9 and 6.5 mas for epochs 1985.5 and 1994.97 respectively, and obtained an averaged rate of separation of $0.27 \mathrm{mas} \mathrm{yr}^{-1}$ from all threeepoch observations (see Table 1 and Fig. 14 of Cotton et al. 1997). A separation of 6.5 mas at epoch 1994.97 is larger than that of 5.76 mas at a later epoch 1997.86 in Table 1. This would be indicative of a back flow in the jet motion, which is very unlikely if not impossible. The 8.6 GHz VLBA observations (Fey \& Charlot 1997) measured a separation of only 5.4 mas at epoch 1995.78, 
which is also much less than that at epoch 1994.97. To further investigate this, we measured the peak-to-peak separation from images directly, and then compared them with those modelfitting results. As a result, we got an excellent agreement between the modelfitted separation and the peak-to-peak separation at epochs 1989.72 and 1997.86 at $5.0 \mathrm{GHz}$ and at epoch 1995.78 at $8.6 \mathrm{GHz}$, but the discrepancy at epochs 1985.5 and 1994.97 was as large as 0.5 and 0.9 mas, respectively. This difference is about one-half and one-quarter of the corresponding beam size, and quite difficult to understand for us at present.

Therefore, we decided to estimate the proper motion by using two epoch (1989.72 and 1997.86) $5 \mathrm{GHz}$ data (denoted by crosses in Fig. 3) only. As shown in Fig. 3, this produces a relative proper motion of 0.093 mas $\mathrm{yr}^{-1}$ between $C_{\mathrm{E}}$ and $C_{\mathrm{W}}$, which corresponds to a superluminal speed of 3.3c. We used other two $5.0 \mathrm{GHz}$ observations to evaluate its uncertainty. The peak-to-peak separation (denoted by open squares in Fig. 3) of 4.4 and 5.6 mas at the corresponding epochs 1985.5 and 1994.97 gave an uncertainty of $5 \%$, while the published points (denoted by filled squares in Fig. 3) would introduce an uncertainty of $16 \%$. This proper motion is much slower than the reported $0.27 \mathrm{mas} \mathrm{yr}^{-1}$ (Cotton et al. 1997). The ongoing monitoring observations confirm that the knot is definitely moving much slower than suggested by the earlier data (Bill Cotton 2000, private communication).

\subsection{Synchrotron self-absorption}

There is a relative position offset of 0.17 mas at epoch 1995.78 for $C_{\mathrm{E}}$ between the position interpolated from $5 \mathrm{GHz}$ data (fitting line in Fig. 3) and the measurement made at $8.6 \mathrm{GHz}$ (Fey \& Charlot 1997) denoted by a open triangle in Fig. 3. Such a frequency dependence of the observed position can be explained by synchrotron selfabsorption (Lobanov 1998). To estimate this position offset using Eq. (11) of Lobanov (1998), we have searched the literature for its radio spectral energy distribution. As a result, the total synchrotron luminosity of $1.510^{45} \mathrm{erg} \mathrm{s}^{-1}$ is estimated. A superluminal speed of 3.3c implies a maximum angle $\theta$ to the line of sight, a minimum jet speed $v(=\beta \mathrm{c})$ and a minimum Lorentz factor $\gamma$ of $34^{\circ}, 0.96 \mathrm{c}$ and 3.5 , respectively. The projected opening angle $\phi_{\mathrm{o}}$ of $5.8^{\circ}$ is measured from $C_{\mathrm{E}}$ component size over its distance to $C_{\mathrm{W}}$ at $8.6 \mathrm{GHz}$. These values predict a maximum position offset of 0.22 mas between 5.0 and $8.6 \mathrm{GHz}$ under the assumption of energy equipartition between radiating particles and magnetic field. The upper limit is mainly due to the uncertainty in the determination of the viewing angle $\theta$ and the resultant lower limits to both $\beta$ and $\gamma$. Thus, such an observed offset in the central region of 3C 138 can be well explained as a frequency dependent shift of the synchrotron self-absorbed core. Assuming a conical jet geometry in which the magnetic field decreases linearly with its distance to the core, we can further derive the magnetic field in the $5 \mathrm{GHz} \mathrm{VLBI}$ core region of
$0.12,0.02$ and $0.01 \mathrm{G}$ for components $C_{\mathrm{W}}, C_{\mathrm{E}}$ and $C_{\mathrm{E} 2}$, respectively (cf. Lobanov 1998).

Recently, a significant position offset has been reported for another superluminal CSS source 3C 216 (see Fig. 3 of Paragi et al. 2000). These may suggest that the core region in CSS sources could also be described by ultracompact jet models, which have been successful in interpreting the central region of radio-loud active galactic nuclei (cf. Lobanov 1998 and references therein).

We are aware, however, that this offset may be due to the positional uncertainties of measurements and limited resolution and blending at lower frequency. Future high resolution, multi-frequency VLBI observations will be critical to clarify this.

\subsection{Core identification}

It is hard to determine the real core in 3C 138 based on the apparent source size and spectral index available now. From their high resolution polarization sensitive imaging, Cotton et al. (1997) have ruled out the possibility that $C_{\mathrm{E}}$ was the core and further suggested that $C_{\mathrm{W}}$ harbors the central energy source. The non variation in the flux density of $C_{\mathrm{E}}$, discussed in Sect. 3.2, can also be viewed as an indication that $C_{\mathrm{E}}$ probably is not related to the center of activity. Future observations with better resolution at lower frequencies (such as the $1.6 \mathrm{GHz}$ space VLBI (VSOP) imaging observations, cf. Hirabayashi et al. 1998) and better sensitivity at higher frequencies should be able to clearly separate and detect the two compact components $C_{\mathrm{W}}$ and $C_{\mathrm{E}}$ and, thus enable us to undoubtedly determine if the spectral index, as suggested by Cotton et al. (1997), is flatter in component $C_{\mathrm{W}}$.

Our new measurements confirm the systematic increasing in the knot separation with time. But the rate is three times slower than the previous one. The detected superluminal speed, when combined with the observed twosided jet structure in $3 \mathrm{C} 138$, poses very strong constraints on the jet kinematics.

The expected ratio of the flux density between jet and counter-jet is given by $R=\left(\frac{1+\beta \cos \theta}{1-\beta \cos \theta}\right)^{2+\alpha}$. Here, intrinsically symmetrical two-sided jets with the same speed in two opposite directions are assumed.

The ratio of the flux density of the eastern to western lobe seen in the $1.7 \mathrm{GHz}$ image (Cotton et al. 1997) is about 16 . Using the spectral index between 1.7 and $5.0 \mathrm{GHz}$ of 0.75 (Fanti et al. 1989), we obtained the product $\beta \cos \theta=0.46$, in good agreement with the value estimated at $5 \mathrm{GHz}$ (Fanti et al. 1989). But this restricts to a maximum apparent speed of $1.6 \mathrm{c}$, which is significantly smaller than 3.3c. Therefore, the superluminal motion of $3.3 \mathrm{c}$ in the central core region, though much less than earlier reported one, is still too high to allow the overall counter-jet structure to be seen in terms of the projection effect and Doppler boosting of an intrinsically identical jet.

One solution to circumvent this is to assume a nonuniform distribution of $R$ for the whole jet since usually 
lobes are observed to move at a much lower speed. This can naturally explain the absence of parsec-scale counterjet in the central region. Cotton et al. (1997) obtained a lower limit to the ratio $R$ of 20 within the central area. Our new observations slightly raised this lower limit to 30 at 5 times rms noise level. These lower limits are consistent with the detected superluminal motion in the inner region being due to the relativistic boosting in the jet.

Such a change in $R$ could be caused by the strong interaction of the jet with the surrounding medium in the immediate region of the core. This is supported by the existence of a large amount of ionized gas in the central region (Cotton et al. 1997). The bending in the jet or a helical jet model is a possible explanation, but unlikely to affect $R$ significantly in case of 3C 138 (cf. Fanti et al. 1989). A superluminal speed of 3.3c implies a ratio $R$ greater than 360 (using the spectral index of component $C_{\mathrm{E}}$ in Table 1), 12 times the lower limit estimated above. On the other hand, the possibility that the observed overall asymmetry is intrinsic is still there, but difficult to test because of the limited data gathered. The nature of the core region is worthy of a more detailed study.

Acknowledgements. We wish to thank Carla Fanti, the referee, for a careful reading of the manuscript and very useful comments, and Bill Cotton for providing us with the $1.7 \mathrm{GHz}$ VLBI image and for communication of information prior to publication. This research has made use of the NASA/IPAC Extragalactic Database (NED) which is operated by the Jet Propulsion Laboratory, Caltech, under contract with the National Aeronautics and Space Administration. This research has made use of data from the University of Michigan
Radio Astronomy Observatory which is supported by the National Science Foundation and by funds from the University of Michigan.

\section{References}

Akujor, C. E., Spencer, R. E., Zhang, F. J., et al. 1993, A\&A, 274,752

Dallacasa, D., Cotton, W. D., Fanti, C., et al. 1995, A\&A, 299, 671

Cotton, W. D., Dallacasa, D., Fanti, C., et al. 1997, A\&A, 325, 493

Fanti, C., Fanti, R., Parma, P., et al. 1989, A\&A, 217, 44

Fanti, R., Fanti, C., Schilizzi, R. T., et al. 1990, A\&A, 231, 333

Fey, A. L., \& Charlot, P. 1997, ApJS, 111, 95

Geldzahler, B. J., Fanti, C., Fanti, R., et al. 1984, A\&A, 131, 232

Hewitt, A., \& Burbidge, G. 1989, ApJS, 69, 1

Hirabayashi, H., Hirosawa, H., Kobayashi, H., et al. 1998, Science, 281, 1825

Kameno, S., Inoue, M., Matsumoto, K., et al. 1995, PASJ, 47, 711

Lobanov, A. P. 1998, A\&A, 330, 79

Lüdke, E., Garrington, S. T., Spencer, R. E., et al. 1998, MNRAS, 299, 467

Nan, R., Cai, Z., Inoue, M., et al. 1992, PASJ, 44, 273

O'Dea, C. P. 1998, PASP, 110, 4930

Paragi, Z., Frey, S., Fejes, I, et al. 2000, PASJ, 52, in press

Piner, B. G., Edwards, P. G., Wehrle, A. E., et al. 2000, ApJ, 537, 91

Schwab, F. R., \& Cotton, W. D. 1983, AJ, 88, 688

Shepherd, M. C. 1997, in Astronomical Data Analysis Software and Systems VI, ed. G. Hunt, \& H. E. Payne, ASP Conf. Ser., 125,77 